\title{
Technical tip: high-resolution isolation of nanoparticle-protein corona complexes from physiological fluids
}

ARTICLE in NANOSCALE · JUNE 2015

Impact Factor: $7.39 \cdot$ DOI: $10.1039 /$ c5nr02618k · Source: PubMed

READS

26

6 AUTHORS, INCLUDING:

Neil Rigby

Institute of Food Research

101 PUBLICATIONS $\quad$ 1,651 CITATIONS

SEE PROFILE

Alan Robert Mackie

Institute of Food Research

168 PUBLICATIONS 3,953 CITATIONS

SEE PROFILE

\section{Balazs Bajka}

Institute of Food Research

11 PUBLICATIONS 128 CITATIONS

SEE PROFILE

Francesca Baldelli Bombelli

Politecnico di Milano

38 PUBLICATIONS 2,003 CITATIONS

SEE PROFILE 


\title{
Nanoscale
}

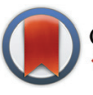

CrossMark \& click for updates

Cite this: Nanoscale, 2015, 7, 11980

\section{Technical tip: high-resolution isolation of nanoparticle-protein corona complexes from physiological fluids $\uparrow$}

\author{
Desirè Di Silvio, ${ }^{\mathrm{a}, \mathrm{b}}$ Neil Rigby, ${ }^{\mathrm{b}}$ Balazs Bajka, ${ }^{\mathrm{b}}$ Andrew Mayes, ${ }^{\mathrm{c}}$ Alan Mackie ${ }^{\mathrm{b}}$ and \\ Francesca Baldelli Bombelli*a,d
}

\begin{abstract}
Nanoparticles (NPs) in contact with biological fluids are generally coated with environmental proteins, forming a stronger layer of proteins around the NP surface called the hard corona. Protein corona complexes provide the biological identity of the NPs and their isolation and characterization are essential to understand their in vitro and in vivo behaviour. Here we present a one-step methodology to recover NPs from complex biological media in a stable non-aggregated form without affecting the structure or composition of the corona. This method allows NPs to be separated from complex fluids containing biological particulates and in a form suitable for use in further experiments. The study has been performed systematically comparing the new proposed methodology to standard approaches for a wide panel of NPs. NPs were first incubated in the biological fluid and successively recovered by sucrose gradient ultracentrifugation in order to separate the NPs and their protein corona from the loosely bound proteins. The isolated NP-protein complexes were characterized by size and protein composition through Dynamic Light Scattering, Nanoparticle Tracking Analysis, SDS-PAGE and LC-MS. The protocol described is versatile and can be applied to diverse nanomaterials and complex fluids. It is shown to have higher resolution in separating the multiple protein corona complexes from a biological environment with a much lower impact on their in situ structure compared to conventional centrifugal approaches.
\end{abstract}

Received 22nd April 2015 Accepted 2nd June 2015

DOI: $10.1039 / \mathrm{c} 5 \mathrm{nr} 02618 \mathrm{k}$

www.rsc.org/nanoscale what is known as a protein corona (PC) ${ }^{11,12}$ This corona provides the biological identity of the NP and determines its interactions with the surrounding biological matter. ${ }^{13,14}$

The PC has a dynamic structure formed by external layers of proteins that quickly exchange with the surrounding environment (soft corona) and an inner layer of proteins strongly bound to the NP surface (hard corona). ${ }^{15-18}$ The PC composition depends on the NP's physical-chemical properties and size, but also the media composition and incubation time have been found to have strong effects. ${ }^{19-21}$ Generally, the hard corona is very stable and almost irreversibly bound to the NP surface when the experimental conditions are kept constant, ${ }^{12}$ although it has recently been shown that small alterations in the composition of the biological media can strongly modify the hard corona. ${ }^{22}$ Moreover, preliminary studies on the evolution of the PC, where NPs were sequentially incubated in different biological fluids, have shown that even if changes occur in the PC upon incubation in the second biological fluid, a sort of fingerprint of the "history" of the NP is kept. ${ }^{23}$ Thus, isolating HC complexes is crucial to independently study their composition and to be able to relate possible biological responses to it.

One of the biggest concerns in this scenario is to isolate HC complexes that preserve the features of those in situ in the bio- 
logical fluid. Commonly, an ex situ approach is used to separate protein-NP complexes from the excess of fluid and isolate hard corona (HC) complexes: sequential cycles of centrifugation/washing are carefully optimized according to the NP and media properties. ${ }^{24,25}$ In many cases this approach is suitable and gives reliable results, but multiple purification steps can alter the equilibrium of the system and lead to corona modifications due to the time scales that characterize the dynamic nature ${ }^{26}$ of the complexes. Application of this approach to NPs characterized by small diameters (about 5-20 nm) and/or low densities (close to $1 \mathrm{~g} \mathrm{~cm}^{-3}$ ) may not achieve a good separation between unbound proteins and PC complexes. High speeds and long times are often necessary with promotion of extensive aggregation with respect to what occurs in situ. These effects are even more accentuated in the isolation of HC complexes for NPs that do not form rich coronas (for example pegylated NPs). For this reason it becomes important to develop methodologies that minimize the number of steps of the ex situ purification of HC complexes to affect as little as possible their properties from in situ.

Furthermore, PC complexes are often very heterogeneous presenting simultaneously monomers, dimers, trimers, etc. for which the actual composition is unknown. In this regard, the biological impact of these co-existing complexes formed by the same NPs might be different as they have different sizes and are likely to carry different proteins. Thus, it is important to be able to separate those different complexes and independently study their effect on the biological matter. Recently, the use of differential sedimentation centrifugation (DCS) permitted the analytical separation of different populations of HC complexes for different NPs and demonstrated that they were representative of those in situ. However, this technique does not allow the recovery of the different populations for further studies. ${ }^{12,27}$ In particular, their recovery becomes important to determine the physical and biological properties of these complexes. Conventional procedures allow the isolation and recovery of mixed PC populations, which are also aggregated with respect to their in situ cognate PC complexes.

Many techniques have been applied to separate and study PC complexes such as size-exclusion chromatography (SEC), magnetic separation through the use of magnetic columns (MACS) able to separate PC complexes of magnetic NPs, ${ }^{28}$ and field-flow-field fractionation. ${ }^{29,30}$ However, none of these methodologies are preparative and permit full recovery of the HC complexes for further studies. Preparative and analytical ultracentrifugation (UC) are widely exploited in biology to isolate cell components and explore protein thermodynamics. ${ }^{31}$ Density gradient UC has been extended to purify NPs from the excess of coating agents, ${ }^{32}$ and obtain narrow size distributions. ${ }^{33,34}$ Recently, Docter, Tenzer and co-workers ${ }^{35,36}$ and Werwie and co-workers ${ }^{37}$ used a sucrose cushion as the first step to separate unbound proteins from corona complexes followed by centrifugal washing to obtain HC complexes.

Here we propose the use of sucrose gradient UC as a onestep methodology to gently separate HC complexes from in situ complex physiological fluids such as bovine serum and diges-



Scheme 1 Scheme of the methodology to isolate and study HC NPs. NPs are incubated in a biological fluid and then subjected to ultracentrifugation (UC). In the image of the vial on the far left, the pink dots are the NPs and the yellow background is the biological medium. In the images of the UC vials (middle images), the green dots represent some proteins that form diverse coronas around the NPs and are separated by UC exploiting density differences.

tive fluids. We have shown that this procedure has a much lower impact on the structure of the complexes and a much higher resolution in separating different complexes with respect to conventional protocols. In fact, this methodology permitted full recovery of HC complexes ex situ minimizing modifications with respect to those in situ and allowing separation of the different populations co-existing in situ (see Scheme 1).

NPs of different size, material and surface coating were tested in serum and in simulated digestive fluids. The PC complexes isolated by conventional centrifugation methods and UC were analysed by size through Nanoparticle Tracking Analysis (NTA) and Dynamic Light Scattering (DLS). The PC composition was investigated by SDS-PAGE and for the PC complexes obtained from digestive fluids, Mass Spectrometry analysis was also performed to confirm the identities of the recovered proteins. Moreover, to prove how the isolation procedure influenced the interaction of the PC complexes with biological matter, the PC complexes of magnetite NPs recovered from serum by both UC and conventional centrifugation methods were incubated on cells in serum-free conditions to compare their NP cellular uptake.

\section{Experimental section}

\section{Materials}

Yellow-green carboxylate-modified polystyrene NPs of $100 \mathrm{~nm}$ and $20 \mathrm{~nm}$ nominal diameter were purchased from Invitrogen (PS-COOH100 and PS-COOH20). PS-COOH100 NPs were pegylated through EDAC chemistry using Jeffamine M1000 (Hunts- 
man $)$ and purified by centrifugal washings $(3 \times 30$ minutes at $17000 \mathrm{rcf}, 20^{\circ} \mathrm{C}$ ) (PS-PEG). Bare $50 \mathrm{~nm}$ silica NPs were purchased from Kisker $\left(\mathrm{SiO}_{2}\right)$. Carboxylated $\mathrm{Fe}_{3} \mathrm{O}_{4}$ NPs were synthesized following the protocol of Sun et al. ${ }^{38}$ and coated by poly(maleic)-alt-1-octadecene (Sigma) according to Lin et al. ${ }^{39}$ obtaining NPs of about $50 \mathrm{~nm}$ in hydrodynamic diameter. For cell uptake studies, $\mathrm{Fe}_{3} \mathrm{O}_{4}$ NPs were fluorescently labelled with BODIPY FLEDA (Lifetechnologies) which was attached to the NP surface by EDAC chemistry. Foetal bovine serum (FBS) was purchased from Fisher. Sucrose, sodium phosphate dibasic, potassium phosphate monobasic, sodium chloride and potassium chloride are from Sigma.

\section{Preparation of the protein corona samples}

NPs in PBS were incubated in 10\%, 55\% and 90\% v/v FBS for one hour at $37{ }^{\circ} \mathrm{C}$. NPs dispersed in FBS solution are called in situ, HC NPs isolated through ultracentrifugation are labelled as UC; HC NPs isolated by a conventional methodology (three centrifugations at $15500 \mathrm{rcf}$ at $4{ }^{\circ} \mathrm{C}$ and re-suspensions in $500 \mu \mathrm{l}$ PBS pH 7.4) are labelled as HC. The samples were characterized by NTA and DLS before and after incubation.

\section{Simulated salivary, gastric and duodenal digestions}

An AT-700 pH Stat Kyoto Electronic Manufacturing Company was used to run simulated digestions. Digestion mixture compositions, time points at which samples were taken and timescales for digestions varied. Buffer solutions were used in all digestions but whilst the desirable $\mathrm{pH}$ was set at the beginning of each phase of digestion, it was not actively controlled throughout (but instead monitored and recorded during the sample taking). The chosen food material was skimmed milk powder (SMP, $34 \mathrm{mg} \mathrm{ml}^{-1}$ ) and individual enzymes in the different digestion phases were used. Pepsin (from porcine gastric mucosa, Sigma-Aldrich, Lot. 091M7020 V) was used in the gastric phase, and trypsin (from porcine pancreas, SigmaAldrich, Lot. 045K7775) and chymotrypsin (from bovine pancreas, Sigma-Aldrich, Lot. 060M7007 V) were used for the duodenal phase, as well as bile. $\mathrm{Fe}_{3} \mathrm{O}_{4}$ NPs at a final concentration of $1.5 \times 10^{13}$ NPs per ml were incubated with $1 \mathrm{ml}$ of fluid collected at time points corresponding to the oral phase $(2 \mathrm{~min}$, $\mathrm{pH} 7$, amylase), before the start of the gastric phase (60 min), after the gastric phase was finished (120 min, $\mathrm{pH} 3$ ) and after duodenal digestion (240 min, $\mathrm{pH}$ 7.0). The NPs were incubated at $37{ }^{\circ} \mathrm{C}$ for one hour in a Brunswick Scientific Excella E24 Incubator Shaker. Equal volumes of purified water were incubated with the fluids to act as controls.

\section{Ultracentrifugation}

Solutions of sucrose were prepared at increasing concentrations and $11 \mathrm{ml}$ of the linear sucrose gradient solutions were layered in $13 \mathrm{ml}$ tubes and left to equilibrate overnight before being subjected to ultracentrifugation using a SW41 Ti rotor (Beckman Coulter) at $20^{\circ} \mathrm{C} .0 .7 \mathrm{ml}$ of the samples was loaded and different protocols were used according to the physical properties of the NP and the biological media (details of the gradient are given in Table 1). After the run, aliquots of $1 \mathrm{ml}$ were
Table 1 Ultracentrifugation experimental conditions used to separate the NPs from the biological fluids. Particles were in PBS, pH 7.4

\begin{tabular}{|c|c|c|c|c|c|}
\hline Type of NP & $\begin{array}{l}\text { Nominal } \\
\text { size }[\mathrm{nm}]\end{array}$ & $\begin{array}{l}\text { NPs } \\
\text { per } \mathrm{ml}^{a}\end{array}$ & $\begin{array}{l}\text { Sucrose } \\
\text { density } \\
\Delta[\% \mathrm{w} / \mathrm{w}]\end{array}$ & $\begin{array}{l}\text { Speed } \\
{[\mathrm{rcf}]}\end{array}$ & $\begin{array}{l}\text { Time } \\
{[\mathrm{min}]}\end{array}$ \\
\hline PS-COOH100 & 100 & $3.6 \times 10^{11}$ & $5-30$ & $77 \mathrm{k}$ & 60 \\
\hline PS-COOH20 & 20 & $4.5 \times 10^{13}$ & $4-40$ & $110 \mathrm{k}$ & 120 \\
\hline PS-PEG & 100 & $3.6 \times 10^{11}$ & $3-30$ & $60 \mathrm{k}$ & 60 \\
\hline $\mathrm{SiO}_{2}$ & 50 & $1.9 \times 10^{13}$ & $3-30$ & $12 \mathrm{k}$ & 20 \\
\hline $\mathrm{Fe}_{3} \mathrm{O}_{4}$ & 50 & $1.5 \times 10^{13}$ & $35-60 / 70$ & $187 \mathrm{k}$ & 120 \\
\hline
\end{tabular}

${ }^{a}$ Concentration of the NPs in the in situ samples before being loaded in the ultracentrifuge.

collected by sucking up sucrose from the top of the tubes to the bottom and analysed to identify protein corona NPs either by screening by dimension or UV absorption at $260 \mathrm{~nm}$.

\section{Dynamic light scattering (DLS)}

Hydrodynamic diameters were measured by Zetasizer SZ (Malvern). $50 \mu \mathrm{l}$ of the samples were diluted in $400 \mu \mathrm{l}$ of PBS in $1 \mathrm{ml}$ cuvettes to obtain attenuation values in the 7-9 range and measured at $25{ }^{\circ} \mathrm{C}$, equilibrating samples for 120 seconds prior to measurement. Data were presented as an average of three measurements. UC samples were dialysed against PBS (2000 MWCO, Spectrum labs) for at least 24 hours at $4{ }^{\circ} \mathrm{C}$ before analysis. The Z-averaged sizes (Z-ave) and the polydispersity index (PdI) were obtained by cumulant analysis of the autocorrelation function.

\section{Nanoparticle tracking analysis (NTA)}

Samples were analysed by NTA by diluting them with MQ water to reach an ideal concentration of $1-3 \times 10^{8}$ NPs per ml. For fluorescently-labelled particles a fluorescence filter ( $\lambda$ of $488 \mathrm{~nm}$ ) was used. Three videos of sixty seconds were collected for each sample and analysed by NTA software. The software is able to track NPs individually and calculates the diffusion coefficient for each one. In this way, a mean value for the hydrodynamic diameter (Mean) is obtained with the relative standard deviation (SD) in respect to all tracks. Results are presented as an average of three independent measurements.

\section{SDS-PAGE electrophoresis}

Dialysed samples were concentrated by Amicon centrifugal filters (MWCO $100 \mathrm{kDa}$, Millipore) at $405 \mathrm{rcf}$ to a final volume of $200 \mu \mathrm{l} .30 \mu \mathrm{l}$ were added to $15 \mu \mathrm{l}$ of SDS-PAGE loading buffer $3 \times\left(10 \%\right.$ DTT, Thermo Scientific) and kept at $98{ }^{\circ} \mathrm{C}$ for 5 minutes. Digested samples that did not contain NPs were mixed in the loading buffer without any previous treatment and denatured as above. HC samples were prepared by resuspending the pellet in $60 \mu \mathrm{l}$ of PBS to which $30 \mu \mathrm{l}$ of the loading buffer was added before denaturation. $20 \mu \mathrm{l}$ of the samples and $5 \mu \mathrm{l}$ of a molecular ladder (Pageruler Broad Range, Biolabs) were loaded in the wells of $12 \%$ Precast Gel NuPAGE (Life Technology). Samples from the simulated digestion were loaded on $10 \%$ Precast Gel NuPAGE (Life Technology) and the 
molecular ladder used was Mark12 Unstained standard from Invitrogen $(5 \mu \mathrm{l})$. The running buffer used was MES buffer (NuPAGE 20×, Invitrogen). Gels were run at $200 \mathrm{~V}$ for 35 minutes. Gels were developed by Sypro Ruby Protein Stain (Biorad) and imaged by Biorad Pharos FX+, the software used to elaborate the images was Image Lab (Biorad).

\section{LC-MS}

A ProPick instrument was used to locate and cut bands from the gel. The bands were digested at $37{ }^{\circ} \mathrm{C}$ for three hours by $10 \mathrm{mM}$ ammonium bicarbonate $10 \mathrm{ng} \mu^{-1}$ Trypsin Gold (Promega, V528A in $50 \mathrm{mM}$ acetic acid) (prepared 01-May-14). $1 \%$ formic acid (Sigma) was added prior to freezing the samples and storing at $-80{ }^{\circ} \mathrm{C}$. Samples were washed in $50 \%$ acetonitrile (Fisher), vortexed and dried out at the Low Drying setting (no heat) on a Speed Vac SC110 (Savant) fitted with a Refrigerated Condensation Trap and a Vac V-500 (Buchi). Samples were again stored at $-80{ }^{\circ} \mathrm{C}$ until ready for Orbitrap analysis. Protein identification was achieved by combining spectrum quality scoring obtained from a conventional database search program MASCOT (Matrix Science, London, England). Search parameters were: peptide mass and fragment mass tolerances of $5 \mathrm{ppm}$ and 0.5 Da respectively, the variable modification was oxidation (M), the fixed modification was carbamidomethyl (C), the enzyme specificity was trypsin, and two missed cleavages were allowed. All of the taxonomy database was searched.

\section{Cell uptake experiments}

M202 cell lines were incubated at $37^{\circ} \mathrm{C}, 5 \% \mathrm{CO} 2 / 95 \%$ air and were cultured in RPMI-1640 (Life Technologies, Carlsbad, CA) supplemented with $10 \%$ heat-inactivated foetal bovine serum (FBS), $2 \mathrm{mM}$ L-glutamine, $100 \mu \mathrm{g} \mathrm{ml}{ }^{-1}$ penicillin and $100 \mu \mathrm{g}$ $\mathrm{ml}^{-1}$ streptomycin all purchased from Life Technologies, Carlsbad, CA. P12 cells were seeded at a density of $4 \times 10^{4}$ cells per ml on glass coverslips and left to adhere overnight. The cells were incubated with $\mathrm{Fe}_{3} \mathrm{O}_{4}$ NPs $\left(10^{12}\right.$ NPs per ml) for 2, 4 and 24 hours. In particular cells were treated with fluorescentlylabelled bare $\mathrm{Fe}_{3} \mathrm{O}_{4}$ NPs, $\mathrm{HC}$, UC1 and UC2. The cells were washed with PBS $(\times 3)$ and fixed (5\% formaldehyde, $2 \%$ sucrose, $0.02 \% \mathrm{w} / \mathrm{v}$ sodium azide in PBS) then permeabilized ( $5 \mathrm{~min}$ with $0.1 \%$ Triton $\mathrm{X}-100$ in PBS). Texas $\operatorname{Red}^{\circledR}-\mathrm{X}$ Phalloidin (Life Technologies) $(6.6 \mu \mathrm{M})$ was employed to stain actin filaments and Hoechst 33342 dye to stain nuclei ( $2 \mu \mathrm{g} \mathrm{ml}{ }^{-1}$, Sigma). The coverslip was mounted face down on a slide for a microscope with $12 \mu \mathrm{l}$ of mounting media (Vectashield, Vector), left to dry overnight and then sealed with nail varnish. A Zeiss LSM510META confocal microscope (UEA) was used to image cells in multi-track mode. ImagePro software was used to elaborate the z-stack recorded. ImagePro software was used to process images.

\section{Results and discussion}

\section{Isolation of hard corona (HC) complexes from FBS}

NPs of different size, material and surface coating (see Table 1) were incubated at $37^{\circ} \mathrm{C}$ in FBS and separated by the excess of proteins by UC on a sucrose gradient. The recovered HC complexes were further characterized and compared with those in situ and those isolated by conventional centrifugation protocols. We chose five different types of NPs that spanned a variety of materials and presented different PC complexes to prove the suitability of this protocol to successfully separate HC complexes of diverse properties. In particular, PS-COOH100 NPs were chosen as the control sample for comparing PC complexes obtained with our novel procedure with those obtained using the conventional approach, ${ }^{12}$ and PS-COOH20 NPs were chosen as an example of NP-protein complexes that cannot be isolated by normal centrifugation, which promoted extensive aggregation and loss of material in the recovery of HC complexes. ${ }^{19}$ PS-PEG NPs were chosen as an example of NPs with a reduced tendency to form a PC in biological environments and thus are very difficult to isolate and recover from the biological fluid, ${ }^{40-42}$ while $\mathrm{SiO}_{2}$ NPs are NPs able to form two populations of HC complexes that could be successfully separated and recovered by UC. ${ }^{27}$ Lastly, $\mathrm{Fe}_{3} \mathrm{O}_{4} \mathrm{NPs}$ are an example of engineered NPs designed for biomedical applications, for which the conventional approaches promoted extensive aggregation. ${ }^{43}$ For all the samples the excess of proteins from the media were enriched in the lower density sucrose layer at the top of the tube as shown in Scheme 1.

The validation of the UC procedure for isolating $\mathrm{HC}$ complexes was first performed by studying $100 \mathrm{~nm}$ PS-COOH NPs dispersed in 90\% FBS, whose PC complexes have already been extensively characterized in the literature. ${ }^{19,44}$ PS-COOH100 in situ was shown to form rather monodisperse PC complexes with a hydrodynamic diameter of about $130 \mathrm{~nm}$ (see Table 2). The isolation of such complexes by normal centrifugation promoted limited aggregation leading to the formation of HC NPs with hydrodynamic diameters of about $170 \mathrm{~nm}$ and characterized by a higher PdI. The same sample subjected to UC produced three separated bands at different concentrations in the sucrose gradient, two of which contained similar complexes and were pooled together. This indicated that with this pro-

Table 2 DLS and NTA characterization of PS-COOH100 NPs in PBS, $90 \% \mathrm{FBS}$, and of $\mathrm{HC}$ complexes isolated by centrifugation $(\mathrm{HC})$ and ultracentrifugation (UC 1-2)

\begin{tabular}{|c|c|c|c|c|c|}
\hline & \multicolumn{2}{|l|}{ DLS } & \multicolumn{3}{|l|}{ NTA } \\
\hline & $D_{\mathrm{H}}^{b}[\mathrm{~nm}]$ & PdI $^{b}$ & $\begin{array}{l}\operatorname{Mean}^{c} \\
{[\mathrm{~nm}]}\end{array}$ & $\begin{array}{l}\mathrm{SD}^{c} \\
{[\mathrm{~nm}]}\end{array}$ & $\mathrm{NPs} / \mathrm{ml}$ \\
\hline $\mathrm{PS}-\mathrm{COOH}^{a}$ & $100.8 \pm 0.7$ & 0.01 & $78 \pm 3$ & $29 \pm 2$ & $7 \times 10^{12}$ \\
\hline PS-COOH in situ & $128.1 \pm 0.9$ & 0.03 & $130 \pm 1$ & $31 \pm 1$ & $3 \times 10^{12}$ \\
\hline PS-COOH HC & $168.0 \pm 0.9$ & 0.26 & $154 \pm 3$ & $52 \pm 7$ & $7 \times 10^{12}$ \\
\hline PS-COOH UC1 & $122.2 \pm 1.7$ & 0.01 & $117 \pm 1$ & $34 \pm 1$ & $5 \times 10^{12}$ \\
\hline PS-COOH UC2 & $131.4 \pm 1.6$ & 0.01 & $126 \pm 2$ & $35 \pm 1$ & $1 \times 10^{12}$ \\
\hline
\end{tabular}

${ }^{a}$ In PBS pH 7.4. ${ }^{b}$ Hydrodynamic diameter $\left(D_{\mathrm{H}}\right)$ and polydispersity index (PdI) obtained by cumulant analysis of the autocorrelation functions. ${ }^{c}$ Mean is the averaged size of all tracked particles and SD is the related Standard Deviation. All the data are presented as the average of three independent measurements. 


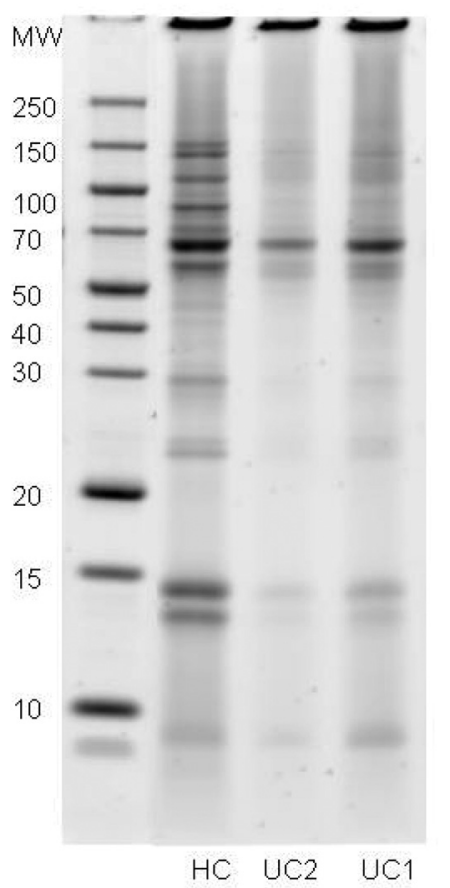

Fig. $1 \mathrm{HC}$ complexes of PS-COOH100. SDS-PAGE gel of the HC complexes of PS-COOH100 NPs in 90\% FBS isolated by conventional centrifugation methods (hard corona, $\mathrm{HC}$ ) and sucrose ultracentrifugation (UC) respectively, as indicated by the label below the tracks of the gel.

cedure it was possible to separate in one-step process HC complexes with a structure similar to those in situ and a high resolution in separation by size. In fact, complexes differing by only $7 \%$ in size (122 nm and $131 \mathrm{~nm}$ ) were isolated in two well-separated sucrose bands (see Fig. 1 UC1 and UC2). The strength of this methodology resides also in the full recovery of the material that could be further analysed to determine the composition of the PC by SDS-PAGE (see Fig. 1) and size (Table 2).

A more challenging task was to recover HC complexes of $20 \mathrm{~nm}$ PS-COOH NPs, for which the conventional centrifugation methodology did not work effectively as it promoted extensive aggregation and loss of material as indicated by the hydrodynamic diameter of about $250 \mathrm{~nm}$ with a PdI of over 0.5 (see Table 3 and Fig. S1 in the ESI $\dagger$ ). In fact, the incubation in $90 \%$ FBS caused the formation of aggregates with an averaged hydrodynamic diameter of $65 \mathrm{~nm}$ compared with $30 \mathrm{~nm}$ of the bare NPs in PBS, and the long times and high speed necessary to sediment the HC complexes clearly amplified this effect. The use of UC allowed us not only to avoid this aggregation, but also to separate the different populations of complexes. In particular, the presence of a small population of large aggregates of about $240 \mathrm{~nm}$ was highlighted. This population was mainly formed by proteins, as indicated by the low density of the sucrose layer (UC1) where they accumulated compared to that of UC2 and UC3, containing most of the complexes. This was also confirmed by the concentrations revealed by NTA for NPs in the UC2 and UC3 sucrose layers, which were comparable to that of the NPs in situ. Moreover, the hydrodynamic
Table 3 DLS and NTA characterization of PS-COOH2O NPs in PBS and $90 \%$ FBS

\begin{tabular}{|c|c|c|c|c|c|}
\hline & \multicolumn{2}{|l|}{ DLS } & \multicolumn{3}{|l|}{ NTA } \\
\hline & $D_{\mathrm{H}}^{b}[\mathrm{~nm}]$ & $\mathrm{PdI}^{b}$ & $\begin{array}{l}\operatorname{Mean}^{c} \\
{[\mathrm{~nm}]}\end{array}$ & $\begin{array}{l}\mathrm{SD}^{c} \\
{[\mathrm{~nm}]}\end{array}$ & $\mathrm{NPs} / \mathrm{ml}$ \\
\hline $\mathrm{PS}-\mathrm{COOH}^{a}$ & $32.5 \pm 0.1$ & 0.10 & $64 \pm 21$ & $28 \pm 5$ & $3 \times 10^{12}$ \\
\hline PS-COOH in situ & $67.6 \pm 0.7$ & 0.31 & $81 \pm 3$ & $38 \pm 6$ & $8 \times 10^{12}$ \\
\hline PS-COOH HC & $247.3 \pm 7.6$ & 0.65 & $186 \pm 18$ & $66 \pm 6$ & $6 \times 10^{11}$ \\
\hline PS-COOH UC1 & $259.5 \pm 4.6$ & 0.43 & $86 \pm 5$ & $59 \pm 4$ & $7 \times 10^{11}$ \\
\hline PS-COOH UC2 & $68.2 \pm 0.1 .9$ & 0.16 & $58 \pm 1$ & $26 \pm 1$ & $1 \times 10^{13}$ \\
\hline PS-COOH UC3 & $91.1 \pm 1.9$ & 0.18 & $73 \pm 3$ & $35 \pm 3$ & $9 \times 10^{12}$ \\
\hline
\end{tabular}

${ }^{a}$ In PBS pH 7.4. ${ }^{b}$ Hydrodynamic diameter $\left(D_{\mathrm{H}}\right)$ and polydispersity index (PdI) obtained by cumulant analysis of the autocorrelation functions. ${ }^{c}$ Mean is the averaged size of all tracked particles and SD is the related Standard Deviation. All the data are presented as the average of three independent measurements.

sizes of the complexes recovered from the UC2 and UC3 layers were comparable to those of the in situ samples and also characterised by a lower PdI related to the separation from the larger protein aggregates as shown in Fig. 2a. The lower sizes detected by NTA for the HC and UC1 samples confirmed the presence of big protein aggregates in these samples, whose lower sizes were likely due to a partial disaggregation by dilution (required for measuring NTA). SDS-PAGE analysis, reported in Fig. $2 b$, showed that fractions UC2 and UC3 were very similar in protein composition, while UC1 was enriched with proteins of $M_{\mathrm{w}}=60-70 \mathrm{kDa}$ and $150 \mathrm{kDa}$ (probably BSA and $\mathrm{IgG})$.

Another case where conventional centrifugation methods have not been very effective in isolating HC complexes is for NPs that do not show a high tendency to adsorb proteins, e.g. pegylated NPs. The difficulty isolating hard corona becomes even greater when these NPs have a low density such as polymeric NPs and liposomes. Ultracentrifugation was used to separate liposomes by $\operatorname{size}^{45}$ and recently Pozzi and coworkers ${ }^{46}$ showed that liposome pegylation could be exploited both to reduce protein adsorption to limit macrophage uptake and to enrich the residual corona with apolipoproteins that bind specifically to some receptors of prostate cancer cells. Pegylated polystyrene NPs (100 nm size) were incubated in 90\% FBS and characterized by DLS and NTA (Table 4). Their dynamic properties did not change significantly with respect to those in PBS indicating a weak interaction with the environmental proteins and no significant changes in the structure of the bare NPs, although protein adsorption could not be completely ruled out. HC complexes isolated by centrifugation were extensively aggregated with respect to those in situ and some loss of material occurred (see Fig. S1 in the ESI $\dagger$ ). The same sample was separated by UC and a well-defined single band enriched in NPs was isolated. The recovered NPs were analysed by DLS and NTA and the results were very similar to those in situ. DLS, unlike NTA, showed an increase in the diameter and PdI with respect to in situ samples (Fig. 3a) and a slight loss of NPs, likely due to the very similar density to the 
(a)
Table 4 DLS and NTA characterization of PS-PEG NPs in different media

\begin{tabular}{|c|c|c|c|c|c|}
\hline & \multicolumn{2}{|l|}{ DLS } & \multicolumn{3}{|l|}{ NTA } \\
\hline & $D_{\mathrm{H}}^{b}[\mathrm{~nm}]$ & PdI $^{b}$ & $\operatorname{Mean}^{c}[\mathrm{~nm}]$ & $\mathrm{SD}^{c}[\mathrm{~nm}]$ & NPs/ml \\
\hline PS-PEG $^{a}$ & $106.3 \pm 0.7$ & 0.04 & $125 \pm 1$ & $38 \pm 1$ & $3 \times 10^{12}$ \\
\hline PS-PEG in situ & $105.9 \pm 0.5$ & 0.10 & $136 \pm 1$ & $42 \pm 2$ & $4 \times 10^{11}$ \\
\hline PES-PEG HC & $155.7 \pm 2.5$ & 0.22 & $123 \pm 10$ & $36 \pm 8$ & $1 \times 10^{11}$ \\
\hline PS-PEG UC & $137.1 \pm 0.1$ & 0.22 & $118 \pm 6$ & $34 \pm 1$ & $7 \times 10^{10}$ \\
\hline
\end{tabular}

${ }^{a}$ In PBS pH 7.4. ${ }^{b}$ Hydrodynamic diameter $\left(D_{\mathrm{H}}\right)$ and polydispersity index (PdI) obtained by cumulant analysis of the autocorrelation functions. ${ }^{c}$ Mean is the averaged size of all tracked particles and SD is the related Standard Deviation. All the data are presented as the

proteins. The corona composition of the complexes isolated by the two methods was compared by SDS-PAGE (see Fig. $3 \mathrm{~b}$ ) and differences could be seen especially for high molecular weight proteins indicating that aggregation and loss of material can ultimately affect the properties of the hard corona of the in situ samples. Thus, UC was shown to be a promising method for recovering HC complexes of pegylated nanomaterials and allows their further characterization and biological response.

Protein coronas of $50 \mathrm{~nm} \mathrm{SiO}_{2}$ NPs have been shown to be very different depending on the protein concentration in the biological fluids, ${ }^{27}$ in particular they form larger aggregates at lower protein concentrations (dimer, trimer, etc.), while smaller and more monodispersed protein-NP complexes form at higher protein concentrations. Here we tried to separate the NP-protein complexes in 10\% FBS (protein concentration average of three independent measurements. (b)

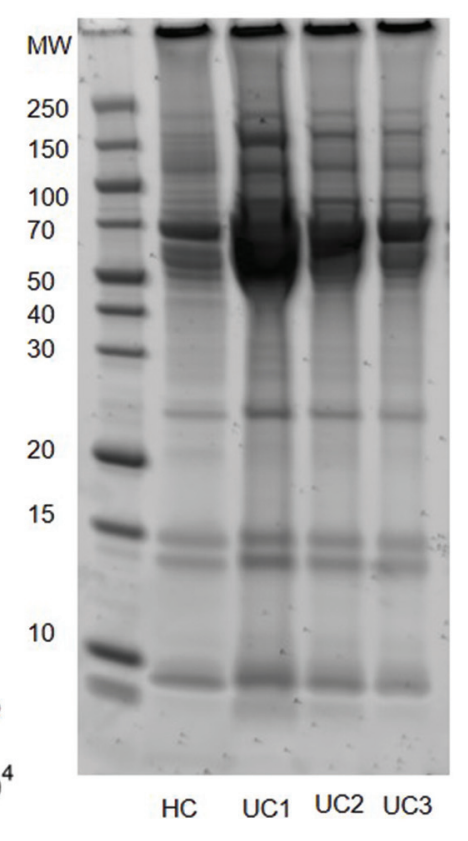

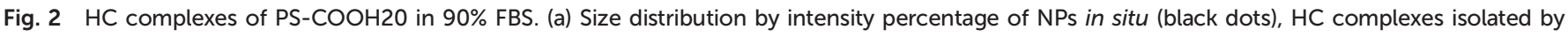
centrifugation (HC, red triangles) and ultracentrifugation (UC2, green empty triangles). (b) SDS-PAGE gel of the complexes in $90 \%$ FBS isolated by conventional centrifugation methods (hard corona, $\mathrm{HC}$ ) and ultracentrifuge (UC1-2), respectively, as indicated by the labels below the tracks of the gel. (a)



(b)

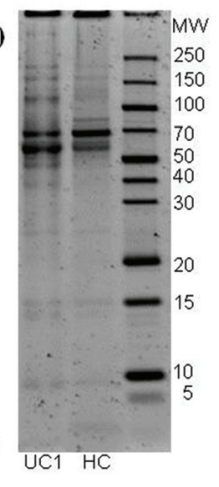

Fig. 3 HC complexes of PS-PEG. (a) Size distribution by intensity percentage of PS-PEG NPs in situ in 90\% FBS (black dots), HC complexes isolated by centrifugation ( $\mathrm{HC}$, red triangles) and ultracentrifugation (UC, green empty triangles). (b) SDS-PAGE gel of the HC complexes of PS-PEG NPs in $90 \%$ FBS isolated by ultracentrifugation (UC) and conventional centrifugation methods $(\mathrm{HC})$, respectively, as indicated by the labels below the tracks of the gel.

$0.0036 \mathrm{~g} \mathrm{ml}^{-1}$ ) in more resolved fractions containing the different species present in situ and compare them with the analogue complexes in 90\% FBS (protein concentration $0.032 \mathrm{~g} \mathrm{ml}^{-1}$ ). Also in this case the formation of protein-NP agglomerates of different size and composition in 10\% FBS made their isolation impractical by conventional centrifugation as extensive aggregation occurred as shown in Table 5. UC resulted in successful separation and recovery of two equivalent (in number of NPs) fractions of NP-protein complexes 
Table 5 DLS and NTA characterization of silica NPs in $10 \%$ and $90 \%$ FBS

\begin{tabular}{|c|c|c|c|c|c|}
\hline & \multicolumn{2}{|l|}{ DLS } & \multicolumn{3}{|l|}{ NTA } \\
\hline & $D_{\mathrm{H}}^{b}[\mathrm{~nm}]$ & $\mathrm{PdI}^{b}$ & $\begin{array}{l}\operatorname{Mean}^{c} \\
{[\mathrm{~nm}]}\end{array}$ & $\begin{array}{l}\mathrm{SD}^{c} \\
{[\mathrm{~nm}]}\end{array}$ & $\mathrm{NPs} / \mathrm{ml}$ \\
\hline $\mathrm{SiO}_{2}{ }^{a}$ & $46.7 \pm 0.5$ & 0.02 & $37 \pm 4$ & $18 \pm 4$ & $6 \times 10^{12}$ \\
\hline $\mathrm{SiO}_{2} 10 \%$ in situ & $141.5 \pm 0.2$ & 0.19 & $211 \pm 13$ & $65 \pm 8$ & $1 \times 10^{13}$ \\
\hline $\mathrm{SiO}_{2} 10 \% \mathrm{HC}$ & $291.0 \pm 3.9$ & 0.36 & $157 \pm 2$ & $73 \pm 1$ & $1 \times 10^{11}$ \\
\hline $\mathrm{SiO}_{2} 10 \%$ UC1 & $123.5 \pm 1.7$ & 0.23 & $98 \pm 3$ & $43 \pm 8$ & $3 \times 10^{10}$ \\
\hline $\mathrm{SiO}_{2} 10 \%$ UC2 & $179.1 \pm 0.9$ & 0.30 & $153 \pm 21$ & $41 \pm 9$ & $4 \times 10^{10}$ \\
\hline $\mathrm{SiO}_{2} 90 \%$ in situ & $81.1 \pm 0.3$ & 0.20 & $108 \pm 5$ & $36 \pm 4$ & $1 \times 10^{13}$ \\
\hline $\mathrm{SiO}_{2} 90 \% \mathrm{HC}$ & $129.7 \pm 4.4$ & 0.27 & $174 \pm 13$ & $75 \pm 15$ & $1 \times 10^{12}$ \\
\hline $\mathrm{SiO}_{2} 90 \% \mathrm{UC}$ & $80.2 \pm 1.1$ & 0.22 & $101 \pm 4$ & $38 \pm 1$ & $9 \times 10^{10}$ \\
\hline
\end{tabular}

${ }^{a}$ In PBS pH 7.4. ${ }^{b}$ Hydrodynamic diameter $\left(D_{\mathrm{H}}\right)$ and polydispersity index (PdI) obtained by cumulant analysis of the autocorrelation functions. ${ }^{c}$ Mean is the averaged size of all tracked particles and SD is the related Standard Deviation. All the data are presented as the average of three independent measurements.

characterized by hydrodynamic diameters of 110 and $180 \mathrm{~nm}$, respectively. SDS-PAGE showed a different pattern of proteins for the two fractions highlighting the different nature of these complexes that are likely to interact differently with biological matter (Fig. 4a). For the samples incubated in 90\% FBS, HC and UC samples showed a very similar protein pattern but DLS and NTA results clearly showed that the NP-protein complexes isolated by UC had a structure more representative of that in situ.

The possibility of isolating those complexes without altering their physical properties is clearly important for studying their biological response in more detail. Fluorescently-labelled water soluble oleic acid coated $\mathrm{Fe}_{3} \mathrm{O}_{4}$ NPs (see Fig. S2-S4 and Table S1 in the ESI $\dagger$ ) coated by PMAO polymer were incubated in 55\% FBS. Larger NP-protein complexes were observed in situ by DLS together with the protein background (peak at 10-15 nm) as shown by the size distribution reported in Fig. 5a. HC samples also showed extensive aggregation and the pellet obtained by centrifugation could not be completely re- (a)

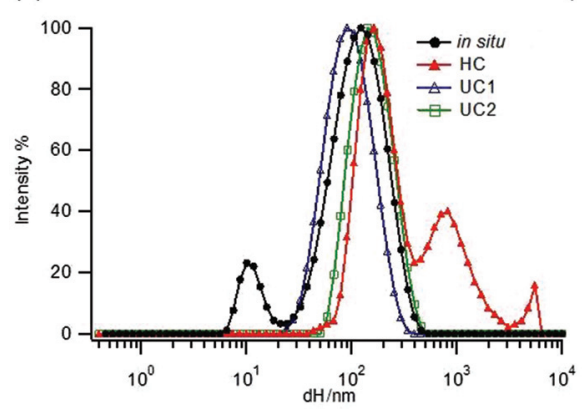

(b) $\mathrm{M}$

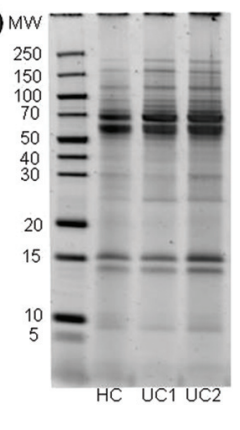

Fig. $5 \mathrm{Fe}_{3} \mathrm{O}_{4}$ in $55 \%$ FBS. (a) Size distributions from DLS measurements of $\mathrm{Fe}_{3} \mathrm{O}_{4} \mathrm{NPs}$ in situ and $\mathrm{HC}$ complexes recovered by ultracentrifugation (UC1-2) and by centrifugal washings (HC). (b) SDS-PAGE of hard corona complexes isolated by conventional centrifugation $(\mathrm{HC})$ and by density gradient ultracentrifugation (UC1-2).

suspended. In contrast, UC samples were successfully isolated and recovered in two NP-protein fractions containing structures of about $77 \mathrm{~nm}$ and $140 \mathrm{~nm}$ in size, respectively, and that seem to correspond to the species present in the in situ sample. The protein patterns were analysed by SDS-PAGE and qualitatively they seemed to be very similar indicating that in this case, $\mathrm{NP}$-protein complexes of different diameters (monomer and dimers) were enriched with similar proteins (Table 6).

$\mathrm{HC}$ complexes recovered by centrifugation and UC were then incubated at a comparable concentration, determined by NTA and fluorescence, in serum-free conditions with M202 cells.

$\mathrm{Fe}_{3} \mathrm{O}_{4}$ NPs were covalently labelled with a fluorescent dye and cells were stained for actin filaments and nuclei. In Fig. 6 representative images of cells incubated for two hours with PC samples in serum-free conditions are reported. A different fluorescent pattern between PC NPs isolated by conventional centrifugation methods (Fig. 6a) and those obtained by UC (Fig. 6b and 6c) can be observed. In fact, the images show that samples treated with HC NPs are characterized by large fluo-

(a)

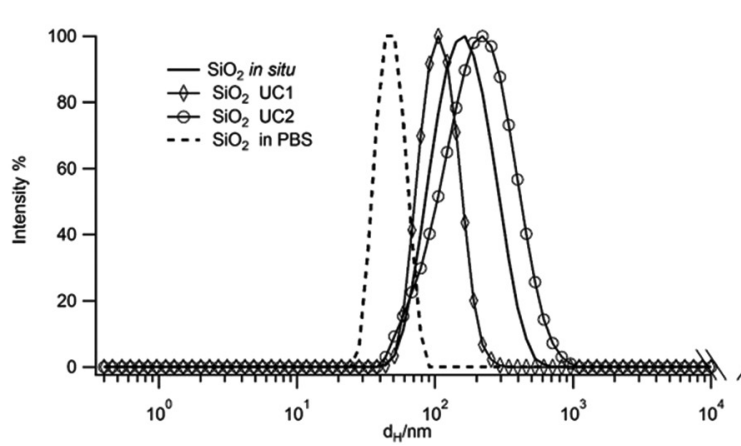

(b)

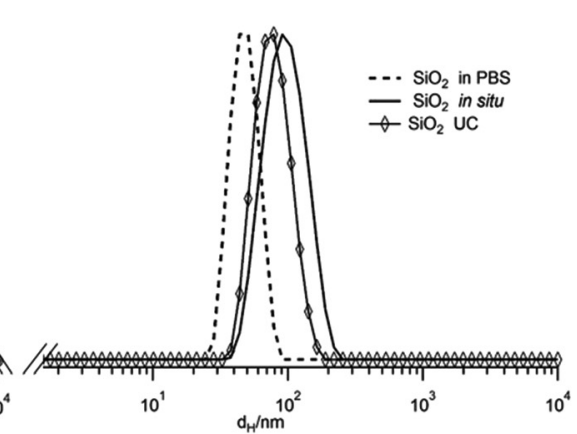

(c)



Fig. 4 Characterization of $\mathrm{SiO}_{2}$ NPs. In graphs (a) and (b) size distributions by intensity percentage of $\mathrm{SiO}_{2} \mathrm{NPs}$ in $10 \%$ and $90 \%$ FBS, respectively, are reported, compared to NPs in PBS (dashed line). (c) SDS-PAGE gel of the hard corona complexes of $\mathrm{SiO}_{2} \mathrm{NPs}$ in $10 \%$ and $90 \%$ FBS isolated by ultracentrifugation $(\mathrm{UC})$ and conventional centrifugation methods $(\mathrm{HC})$, respectively, as indicated by the labels below the tracks of the gel. 
Table 6 DLS and NTA characterization of $\mathrm{Fe}_{3} \mathrm{O}_{4}$ NPs in 55\% FBS

\begin{tabular}{lrllll}
\hline & \multicolumn{1}{l}{$\mathrm{DLS}$} & & $\mathrm{NTA}$ & \\
\cline { 2 - 3 } \cline { 6 - 6 } & \multicolumn{1}{l}{$D_{\mathrm{H}}{ }^{b}[\mathrm{~nm}]$} & $\mathrm{PdI}^{b}$ & & $\operatorname{Mean}^{c}[\mathrm{~nm}]$ & $\mathrm{SD}^{c}[\mathrm{~nm}]$ \\
\hline $\mathrm{Fe}_{3} \mathrm{O}_{4}{ }^{a}$ & $51.4 \pm 0.3$ & 0.17 & & $93 \pm 7$ & $42 \pm 12$ \\
$\mathrm{Fe}_{3} \mathrm{O}_{4}$ in situ & $63.1 \pm 1.8$ & 0.50 & & $157 \pm 3$ & $51 \pm 4$ \\
$\mathrm{Fe}_{3} \mathrm{O}_{4} \mathrm{HC}^{d}$ & $251.8 \pm 9.5$ & 0.55 & & n.d. & n.d. \\
$\mathrm{Fe}_{3} \mathrm{O}_{4}$ UC1 & $77.4 \pm 0.2$ & 0.26 & & $90 \pm 1$ & $33 \pm 1$ \\
$\mathrm{Fe}_{3} \mathrm{O}_{4}$ UC2 & $147.1 \pm 4.3$ & 0.20 & & $139 \pm 3$ & $47 \pm 2$
\end{tabular}

${ }^{a}$ in PBS pH 7.4. ${ }^{b}$ Hydrodynamic diameter $\left(D_{\mathrm{H}}\right)$ and polydispersity index (PdI) obtained by cumulant analysis of the autocorrelation functions. ${ }^{c}$ Mean is the averaged size of all tracked particles and SD is the related Standard Deviation. All the data are presented as the average of three independent measurements. ${ }^{d}$ Sample too polydispersed to be measured.

rescent aggregates hardly taken up by the cells, while those treated with the PC complexes from UC fractions are more monodispersed in size and showed a much higher cell uptake. No significant differences are instead observed in NP cell uptake from the two UC fractions indicating that, in this case, differently structured PC complexes are "seen" similarly by the cells. This is also in agreement with the protein corona pattern observed in the SDS-PAGE (see Fig. 5). Uptake experiments done at different times of incubation, $4 \mathrm{~h}$ and $24 \mathrm{~h}$, did not show major changes (data not shown).

\section{Recovery of HC complexes from gastrointestinal fluids}

The importance of this methodology has also been demonstrated in the recovery of NP-protein complexes from different non-serum biological fluids such as simulated gastrointestinal fluids. The digestion of NPs in these fluids required a very long and complex protocol (see Materials and methods sections) for which the isolation with conventional methods was ineffective, due to the NP concentration and agglomeration problems. Investigation of the protein coronas of NPs in gastrointestinal fluids is important in relation to the appli- cation of NPs in food, ${ }^{8}$ food packaging, toxicology ${ }^{4-49}$ and medicine. It has been shown that NPs such as iron oxide and $\mathrm{Ag}$ NPs tend to aggregate in the gastrointestinal tract $^{50,51}$ because of the extreme conditions of ionic strength and $\mathrm{pH}$. In a recent paper, Seung-Chul Yang and co-workers ${ }^{52}$ proposed a procedure to stabilize iron oxide NPs in aqueous solution and highlighted that in digestive fluids aggregation occurred, but primary particle size measurements are still possible if factors affecting colloidal stability such as enzymes, $\mathrm{pH}$ and electrolytes were removed from samples.

In this scenario, density gradient UC is the appropriate technique to extract PC complexes from these fluids without over-manipulating the samples. In particular, we incubated $\mathrm{Fe}_{3} \mathrm{O}_{4}$ NPs in simulated salivary, gastric and intestinal fluids prepared as described in the Materials and methods sections. NPs appeared to be stable in salivary and gastric fluids forming small clusters of about $100 \mathrm{~nm}$ (see Fig. 5 and 6 in the ESI $\dagger$ ), while extensive aggregation occurred in the intestinal conditions. In saliva and gastric fluids the recovery and analysis of PC complexes was also possible with conventional methods, although their protein composition was likely affected with respect to that in situ. In fact, although NTA showed very similar distributions for corona complexes isolated by UC compared to those by the normal protocol (Fig. 7a), the protein patterns, shown in the SDS-PAGE, were different (Fig. 7b) indicating a strong enrichment of proteins in the $\mathrm{HC}$ samples probably due to contamination from protein agglomerates that sediment together with the PC complexes. These aggregates are instead removed in the first sucrose gradient layers by UC with the recovery of pure fractions with PC complexes. Subsequent LC-MS analysis of the PC complexes isolated by UC showed that they were mainly composed of pepsin (34 kDa), some selected peptides from $\beta$-casein hydrolysis at a very low molecular weight $(6 \mathrm{kDa}),{ }^{53,54}$ $\alpha$-lactalbumin (14.4 kDa) and $\beta$-lactoglobulin (18.4 kDa), while HC fractions were characterized by some stronger bands at a high molecular weight and consisted of a number of hydrolysed fragments not easily identifiable ${ }^{55}$ probably due to an
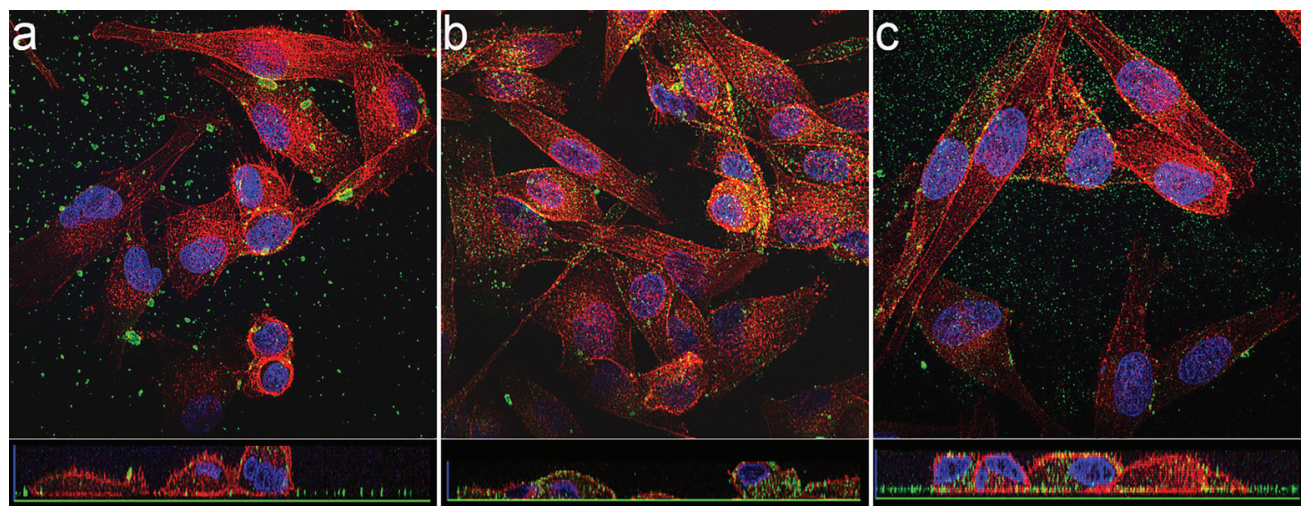

Fig. $6 \mathrm{Fe}_{3} \mathrm{O}_{4} \mathrm{PC}$ uptake. Confocal images of M202 cells after 2 hours of incubation with $\mathrm{Fe}_{3} \mathrm{O}_{4} \mathrm{PC}$ complexes $\left(10^{12} \mathrm{NPs}\right.$ per ml) isolated a) by centrifugation ( $\mathrm{HC}$ ), and (b) and (c) by ultracentrifugation. Actin filaments were stained by Texas Red ${ }^{\circledR}-\mathrm{X}$ Phalloidin (red channel), nuclei by Hoechst 33342 (blue channel) and NPs by BODIPY FL EDA (green channel). 
(a)

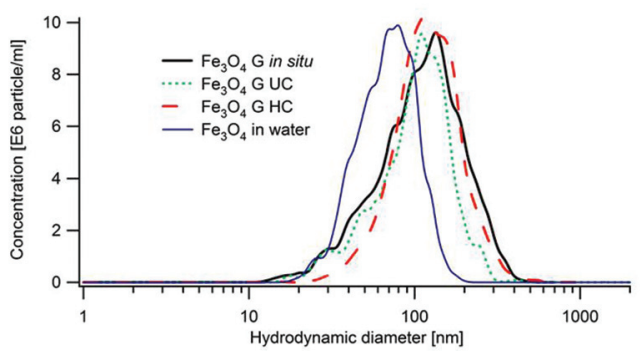

(b)

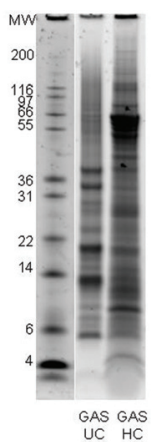

Fig. $7 \quad \mathrm{Fe}_{3} \mathrm{O}_{4}$ NPs in simulated gastric fluid. (a) Size distributions obtained from NTA for $\mathrm{Fe}_{3} \mathrm{O}_{4}$ NPs incubated with gastric fluids and the relative hard coronas isolated by ultracentrifugation (UC) and centrifugation (HC). (b) SDS-PAGE of the hard corona samples isolated by ultracentrifugation (UC) and conventional centrifugation (HC) methods, respectively, as indicated by the labels below the tracks of the gel.

extended exposure time with the biological fluid during the pellet procedure in the centrifuge (see the $\mathrm{ESI} \dagger$ for LC-MS data). ${ }^{35,56,57}$

In contrast, the extensive aggregation combined with the complex composition of the intestinal media made it impossible to extract the PC complexes from this environment by conventional methods. Mainly composed of enzymes (trypsin, $23 \mathrm{kDa}$, and chymotrypsin, $25 \mathrm{kDa}$ ) and some persistent peptides, the intestinal environment led to strong NP aggregation but the PC complexes could be isolated through UC. NTA on the starting sample (in situ) showed a wide range of particulates ranging from $200 \mathrm{~nm}$ to micron size. NTA on UC fractions containing PC complexes recovered from this environment showed a small and relatively narrow population without contamination of the larger complexes that were instead found in the HC complexes isolated by centrifugal washings (see Fig. 8).

To rule out the possibility of proteins running through the sucrose gradient, control gels were performed with fluids in the absence of NPs as can be seen in Fig. 9. The first two sucrose fractions of all samples (with and without NPs) contained the unbound proteins, showing a similar composition in both samples (lanes $1-2$, Fig. 9a and lanes $1^{*}-2^{*}$, Fig. 9b). In the sample with the NPs the sucrose layer labelled as 8 showed the presence of a rich protein pattern with respect to the control that did not show the presence of any protein (lane $8^{*}$, Fig. 9c). In fact, sucrose fraction 8 was also brown coloured indicating the presence of $\mathrm{Fe}_{3} \mathrm{O}_{4}$ NPs. The hard and soft coronas obtained by centrifugation are very similar (lane $\mathrm{HC}$ and SC of Fig. 9a) while the corona isolated by UC (lane 8, Fig. 9a) presented some notable bands at $21 \mathrm{kDa}$ persistent from the gastric phase and chymotrypsin at $25 \mathrm{kDa}$. Bile salts caused desorption of proteins according to their concentration and exposure time ${ }^{35,58,59}$ therefore also in this case the ability of UC to limit the contact time between the PC complexes and biological medium may affect the corona composition. Some bands at a higher molecular weight could not be found anywhere else and showed quite regular spacing among them.

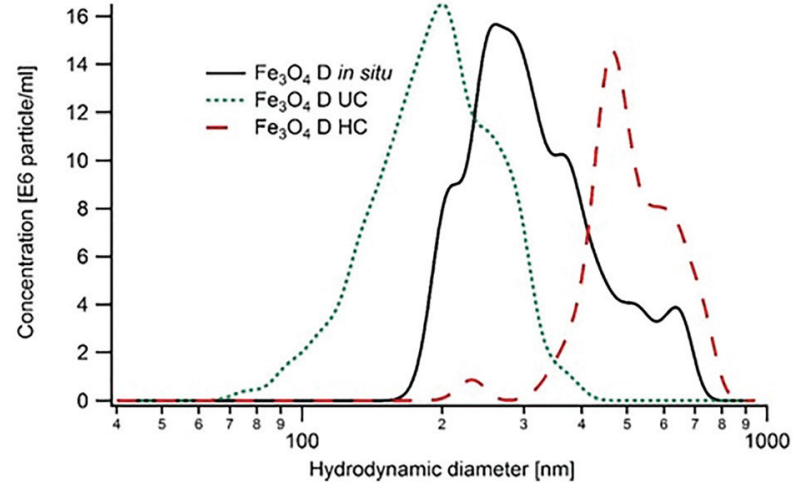

Fig. $8 \mathrm{Fe}_{3} \mathrm{O}_{4}$ NPs in simulated intestinal fluid. Size distributions obtained from NTA for $\mathrm{Fe}_{3} \mathrm{O}_{4}$ NPs incubated with intestinal fluids for one hour and the relative hard coronas isolated by ultracentrifugation (UC) and centrifugation (HC).

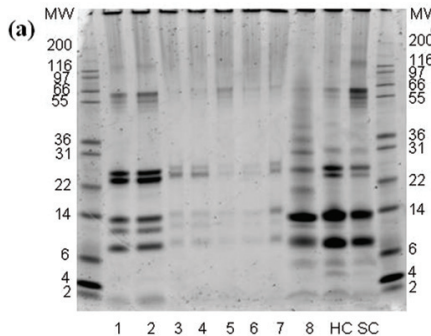

(c)
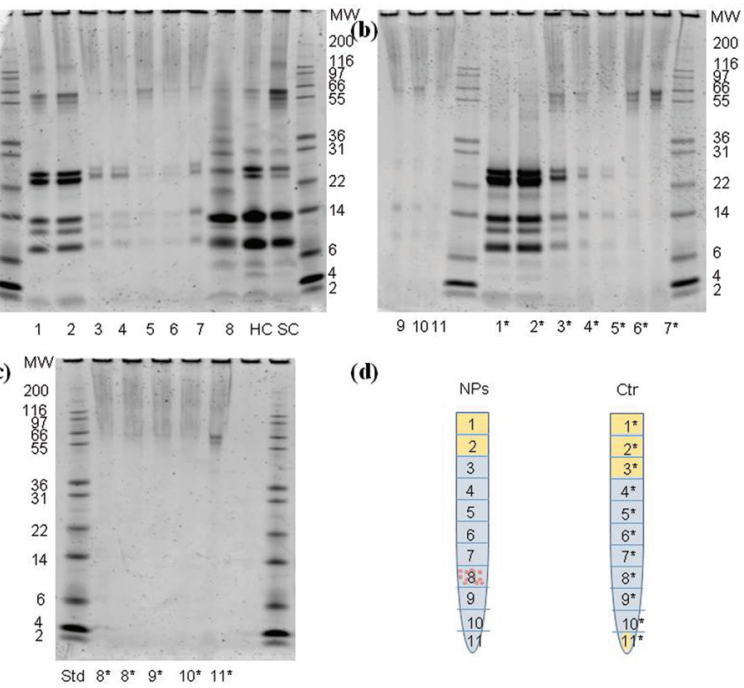

(d)

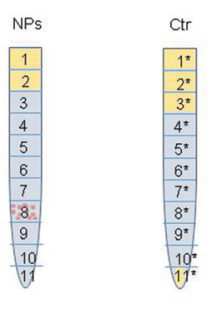

Fig. 9 SDS-PAGE of $\mathrm{Fe}_{3} \mathrm{O}_{4}$ NPs in simulated intestinal fluid. (a) SDS-gel containing UC fractions of the sample of intestinal fluid containing the NPs. Lane 8 contains the PC complexes, and the lanes called HC and SC contain soft corona and hard corona complexes, respectively, isolated by conventional methods. (b) and (c) Control samples without NPs to show no contamination from free proteins in the lanes with the PC complexes. (d) Schematic drawing of the sucrose layer arrangement in the UC tube showing where the NPs and proteins were found in the samples with NPs (NPs) and without NPs (Ctr).

From molecular weight estimation, we assume these to be oligomers of a very small fragment $(4.9 \mathrm{kDa})$ although lower weight oligomers seem to be missing.

\section{Conclusions}

In this study we demonstrated that sucrose gradient ultracentrifugation is an effective tool in the isolation of protein corona complexes from complex biological media without 
affecting their structure and composition with respect to those in situ. This approach can be applied to a wide range of nanoparticles by simply tuning the experimental conditions (centrifugation rate, time, temperature). Moreover, it proved to be a less invasive method keeping the structure and composition of the PC complexes intact as well as having a much higher resolution compared to conventional approaches in terms of separation by size. In fact, not only could the NPs be fully recovered from the environment, but also the structure of the resulting NP-protein complexes was more representative of that of the complexes in situ. Isolated PC complexes were also incubated with cells and those recovered by the UC methodology showed much less aggregation and a higher uptake with respect to those recovered by conventional methods. Moreover, it also allowed a fine separation of the different protein-NP aggregates to be present simultaneously in the biological environment even from very complex matrices such as simulated digestive fluids. In fact, to the best of our knowledge, this is the first example of the recovery of PC complexes from digestive fluids, in which NPs are known to aggregate extensively (mostly in intestinal conditions).

The ability to isolate and recover different NP-protein complexes in a stable form with such a high size resolution from diverse biological media may have a significant impact on the interpretation of the role of the protein corona in the interaction with cellular mechanisms. However, the biological relevance of these different complexes remains to be investigated.

\section{Acknowledgements}

This work was supported by funds from the British Skin Foundation and the Royal Society. FBB thanks the financial support from Regione Lombardia (Fondo per lo Sviluppo e la Coesione - FAS 2007-2013). Dr Davide Carta and Procarta are acknowledged for their help with NTA measurements. We thank Francesco Rossi for the preparation of digestive fluids.

\section{Notes and references}

1 D. Mishra, J. R. Hubenak and A. B. Mathur, J. Biomed. Mater. Res., Part A, 2013, 101, 3646-3660.

2 M. L. Etheridge, S. A. Campbell, A. G. Erdman, C. L. Haynes, S. M. Wolf and J. McCullough, Nanomedicine, 2013, 9, 1-14.

3 E. W. Group, Nano-materials: prevalence in personal care products, http://www.ewg.org/skindeep/2007/08/25/hundreds-of-personal-care-products-contain-poorly-studiednano-materials/.

4 J. P. Kaiser, S. Zuin and P. Wick, Sci. Total Environ., 2013, 442, 282-289.

5 W. Zheng, P. Huang, D. Tu, E. Ma, H. Zhu and X. Chen, Chem. Soc. Rev., 2015, 44(6), 1379-1415.

6 Z. Valerio, A. Gabriele De, V. Luigi, C. Valentina, M. Claudia, G. Jacek, R. Andrea, C. Aldo Di and M. B. Thomas, Nanotechnology, 2013, 24, 255401.
7 A. N. Shipway, E. Katz and I. Willner, ChemPhysChem, 2000, 1, 18-52.

8 T. Borel and C. M. Sabliov, Annu. Rev. Food Sci. Technol., 2014, 5, 197-213.

9 N. Lewinski, V. Colvin and R. Drezek, Small, 2008, 4, 2649.

10 A. E. Nel, L. Madler, D. Velegol, T. Xia, E. M. V. Hoek, P. Somasundaran, F. Klaessig, V. Castranova and M. Thompson, Nat. Mater., 2009, 8, 543-557.

11 T. Cedervall, I. Lynch, S. Lindman, T. Berggård, E. Thulin, H. Nilsson, K. A. Dawson and S. Linse, Proc. Natl. Acad. Sci. U. S. A., 2007, 104, 2050-2055.

12 D. Walczyk, F. Baldelli Bombelli, M. P. Monopoli, I. Lynch and K. A. Dawson, J. Am. Chem. Soc., 2010, 132, 5761-5768.

13 M. Mahmoudi, J. Meng, X. Xue, X. J. Liang, M. Rahman, C. Pfeiffer, R. Hartmann, P. R. Gil, B. Pelaz, W. J. Parak, P. del Pino, S. Carregal-Romero, A. G. Kanaras and S. Tamil Selvan, Biotechnol. Adv., 2014, 32, 679-692.

14 C. D. Walkey and W. C. W. Chan, Chem. Soc. Rev., 2012, 41, 2780-2799.

15 I. Lynch and K. A. Dawson, Nano Today, 2008, 3, 40-47.

16 S. Milani, F. Baldelli Bombelli, A. S. Pitek, K. A. Dawson and J. Rädler, ACS Nano, 2012, 6, 2532-2541.

17 J. Wang, U. B. Jensen, G. V. Jensen, S. Shipovskov, V. S. Balakrishnan, D. Otzen, J. S. Pedersen, F. Besenbacher and D. S. Sutherland, Nano Lett., 2011, 11, 4985-4991.

18 E. A. Vogler, Biomaterials, 2012, 33, 1201-1237.

19 M. Lundqvist, J. Stigler, G. Elia, I. Lynch, T. Cedervall and K. A. Dawson, Proc. Natl. Acad. Sci. U. S. A., 2008, 105, 14265-14270.

20 E. Casals, T. Pfaller, A. Duschl, G. J. Oostingh and V. Puntes, ACS Nano, 2010, 4, 3623-3632.

21 W. G. Kreyling, S. Fertsch-Gapp, M. Schäffler, B. D. Johnston, N. Haberl, C. Pfeiffer, J. Diendorf, C. Schleh, S. Hirn, M. Semmler-Behnke, M. Epple and W. J. Parak, Beilstein J. Nanotechnol., 2014, 5, 16991711.

22 A. Albanese, C. D. Walkey, J. B. Olsen, H. Guo, A. Emili and W. C. W. Chan, ACS Nano, 2014, 8, 5515-5526.

23 M. Lundqvist, J. Stigler, T. Cedervall, T. Berggård, M. B. Flanagan, I. Lynch, G. Elia and K. Dawson, ACS Nano, 2011, 5, 7503-7509.

24 V. Sherwood, D. Di Silvio and F. Baldelli Bombelli, Nanoscopic Agents in a Physiological Environment: The Importance of Understanding Their Characteristics, Springer, Berlin Heidelberg, 2014, ch. 36, pp. 1-26.

25 S. R. Saptarshi, A. L. Duschl A Fau - Lopata and A. L. Lopata, J. Nanobiotechnol., 2013, 11, 26.

26 P. d. Pino, B. Pelaz, Q. Zhang, P. Maffre, G. U. Nienhaus and W. J. Parak, Mater. Horiz., 2014, 1, 301-313.

27 M. P. Monopoli, D. Walczyk, A. Campbell, G. Elia, I. Lynch, F. Baldelli Bombelli and K. A. Dawson, J. Am. Chem. Soc., 2011, 133, 2525-2534.

28 U. Sakulkhu, M. Mahmoudi, L. Maurizi, J. Salaklang and H. Hofmann, Sci. Rep., 2014, 4. 
29 L. Böhmert, M. Girod, U. Hansen, R. Maul, P. Knappe, B. Niemann, S. M. Weidner, A. F. Thünemann and A. Lampen, Nanotoxicology, 2013, 8, 631-642.

30 J. Ashby, S. Schachermeyer, S. Pan and W. Zhong, Anal. Chem., 2013, 85, 7494-7501.

31 G. J. Howlett, A. P. Minton and G. Rivas, Curr. Opin. Chem. Biol., 2006, 10, 430-436.

32 K. L. Planken and H. Colfen, Nanoscale, 2010, 2, 18491869.

33 N. Kanokwan, R. Vinayak, A. F. Jeffrey and R. Vytas, Nanotechnology, 2013, 24, 155701.

34 K. E. Moore, M. Pfohl, F. Hennrich, V. S. K. Chakradhanula, C. Kuebel, M. M. Kappes, J. G. Shapter, R. Krupke and B. S. Flavel, ACS Nano, 2014, 8, 6756-6764.

35 D. Docter, U. Distler, W. Storck, J. Kuharev, D. Wünsch, A. Hahlbrock, S. K. Knauer, S. Tenzer and R. H. Stauber, Nat. Protocols, 2014, 9, 2030-2044.

36 S. Tenzer, D. Docter, J. Kuharev, A. Musyanovych, V. Fetz, R. Hecht, F. Schlenk, D. Fischer, K. Kiouptsi, C. Reinhardt, K. Landfester, H. Schild, M. Maskos, S. K. Knauer and R. H. Stauber, Nat. Nanotechnol., 2013, 8, 772-781.

37 M. Werwie, N. Fehr, X. Xu, T. Basché and H. Paulsen, Biochim. Biophys. Acta, 2014, 1840, 1651-1656.

38 S. Sun, H. Zeng, D. B. Robinson, S. Raoux, P. M. Rice, S. X. Wang and G. Li, J. Am. Chem. Soc., 2003, 126, 273-279.

39 C.-A. J. Lin, R. A. Sperling, J. K. Li, T.-Y. Yang, P.-Y. Li, M. Zanella, W. H. Chang and W. J. Parak, Small, 2008, 4, 334-341.

40 S. Stolnik, S. Dunn, M. Garnett, M. Davies, A. A. Coombes, D. C. Taylor, M. P. Irving, S. C. Purkiss, T. F. Tadros, S. Davis and L. Illum, Pharm. Res., 1994, 11, 1800-1808.

41 J.-C. Leroux, P. Gravel, L. Balant, B. Volet, B. M. Anner, E. Allémann, E. Doelker and R. Gurny, J. Biomed. Mater. Res., 1994, 28, 471-481.

42 V. Torrisi, A. Graillot, L. Vitorazi, Q. Crouzet, G. Marletta, C. Loubat and J. F. Berret, Biomacromolecules, 2014, 15, 3171-3179.

43 M. Mahmoudi, M. A. Shokrgozar, S. Sardari, M. K. Moghadam, H. Vali, S. Laurent and P. Stroeve, Nanoscale, 2011, 3, 1127-1138.
44 M. Jansch, P. Stumpf, C. Graf, E. Rühl and R. H. Müller, Int. J. Pharm., 2012, 428, 125-133.

45 V. Sánchez-López, J. M. Fernández-Romero and A. GómezHens, Anal. Chim. Acta, 2009, 645, 79-85.

46 D. Pozzi, V. Colapicchioni, G. Caracciolo, S. Piovesana, A. L. Capriotti, S. Palchetti, S. De Grossi, A. Riccioli, H. Amenitsch and A. Lagana, Nanoscale, 2014, 6, 27822792.

47 G. Maiorano, S. Sabella, B. Sorce, V. Brunetti, M. A. Malvindi, R. Cingolani and P. P. Pompa, ACS Nano, 2010, 4, 7481-7491.

48 M. J. D. Clift, S. Bhattacharjee, D. M. Brown and V. Stone, Toxicol. Lett., 2010, 198, 358-365.

49 Q. Peng, S. Zhang, Q. Yang, T. Zhang, X.-Q. Wei, L. Jiang, C.-L. Zhang, Q.-M. Chen, Z.-R. Zhang and Y.-F. Lin, Biomaterials, 2013, 34, 8521-8530.

50 E. Zimmermann and R. H. Müller, Eur. J. Pharm. Biopharm., 2001, 52, 203-210.

51 Y. V. Frenkel, A. D. Clark, K. Das, Y.-H. Wang, P. J. Lewi, P. A. J. Janssen and E. Arnold, J. Med. Chem., 2005, 48, 1974-1983.

52 S.-C. Yang, S.-Y.-R. Paik, J. Ryu, K.-O. Choi, T. S. Kang, J. K. Lee, C. W. Song and S. Ko, Food Chem., 2014, 161, 185-191.

53 A. Macierzanka, A. I. Sancho, E. N. C. Mills, N. M. Rigby and A. R. Mackie, Soft Matter, 2009, 5, 538-550.

54 M. Defernez, G. Mandalari and E. N. C. Mills, Electrophoresis, 2010, 31, 2838-2848.

55 N. Kitabatake and Y.-I. Kinekawa, J. Agric. Food Chem., 1998, 46, 4917-4923.

56 M. P. Monopoli, C. Aberg, A. Salvati and K. A. Dawson, Nat. Nanotechnol., 2012, 7, 779-786.

57 E. Monogioudi, G. Faccio, M. Lille, K. Poutanen, J. Buchert and M.-L. Mattinen, Food Hydrocolloids, 2011, 25, 71-81.

58 T. Winuprasith, S. Chantarak, M. Suphantharika, L. He and D. J. McClements, J. Colloid Interface Sci., 2014, 426, 333340 .

59 J. Maldonado-Valderrama, N. C. Woodward, A. P. Gunning, M. J. Ridout, F. A. Husband, A. R. Mackie, V. J. Morris and P. J. Wilde, Langmuir, 2008, 24, 6759-6767. 